DOI: http://dx.doi.org/10.33846/hn50305

http://heanoti.com/index.php/hn

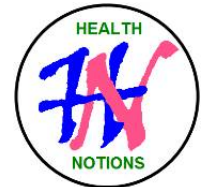

RESEARCH ARTICLE

URL of this article: http://heanoti.com/index.php/hn/article/view/hn50305

\title{
Characteristics of Acute Kidney Injury in the Intensive Care Unit of Dr. Soetomo
} General Hospital, Surabaya

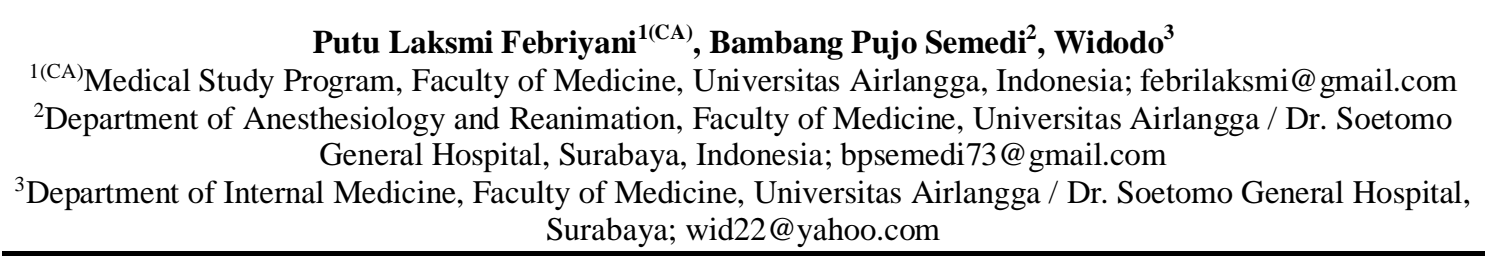

\section{ABSTRACT}

Acute Kidney Injury (AKI) is one of the causes of morbidity and mortality among people in both developing and developed countries in the intensive care unit (ICU). In Indonesia, the incidence rate is $0.2 \%$ while for the Surabaya, AKI research has not been widely carried out and published. This study aims to determine the characteristics of AKI patients in ICU of Dr. Soetomo hospital covers the data distribution of sociodemographic, risk factors, and mortality. This descriptive study involving 23 inpatients at the ICU with AKI as the sample. Samples were selected using total sampling method. Data were collected from medical records with data collection sheets. The results were then analyzed descriptively and tested bivariate using logistic regression. The results showed that $82.6 \%$ of the patients were male with the highest age range of 50-56 years old (30.43\%). AKI mortality rate in the intensive care unit was $30.43 \%$. The primary diagnosis was diabetes mellitus $(34.78 \%)$ and the highest exposure factor was a septic shock (38.70\%). Factors associated with AKI mortality were diabetes mellitus $(\mathrm{p}=0.000)$ and exposure to septic shock $(\mathrm{p}=0.005)$.

Keywords: acute kidney injury; intensive care unit

\section{INTRODUCTION}

Acute Kidney Injury (AKI) is a sudden decrease in kidney function characterized by a decrease in glomerular filtration rate (GFR), an increase in serum creatinine, and a decrease in urine volume excretion from the body. This disorder can occur due to prerenal, intrarenal, and postrenal factors ${ }^{(1)}$. In the last 15 years, the incidence of AKI has shown an increase. The incidence of MMR in hospitals in developed countries that are reported to be higher than in developing countries may be related to incomplete recording and reporting. Reports in developed countries state that the incidence of AKI in the community is $0.5 \%-0.9 \%$, in patients hospitalized is $0.7 \%-18 \%$, patients treated in the ICU are $36 \%-67 \%$ with the AKI mortality rate ranging from $25 \%$ to $80 \%{ }^{(2)}$. In a study conducted by Harrois, AKI was the third leading cause of death after bleeding and concussion in the intensive care $u_{n i t}{ }^{(3)}$. The incidence of the Indonesian population suffering from acute kidney problems is $0.2 \%$ or 2 per 1000 population.

In a study conducted by Venot in 2015 in an intensive care unit, AKI patients due to sepsis had the highest mortality association, $70 \%$, and the use of renal replacement therapy was more in AKI with diabetic comorbidities than non-diabetics, $67 \%$ versus $32 \%{ }^{(4)}$. A study conducted by Patschan in 2016, showed as many as $33 \%$ of AKI patients undergoing renal replacement therapy had comorbidities with diabetes as the highest comorbid ${ }^{(5)}$. In Surabaya, there is no data or research on AKI cases in the intensive care unit. Therefore, the authors are very interested in studying the characteristics of AKI in the intensive care unit of Dr. Soetomo Hospital as an initial description.

\section{METHODS}

By looking at the occurrence of MMR that occurred in the ICU at Dr. Soetomo Hospital, Surabaya, this study used a descriptive study. The study was conducted in the ICU room of Dr. Soetomo Hospital in August - November 2019.

The study sample was taken by total sampling, including as many as 23 individuals among all inpatients with an AKI diagnosis. Data analysis carried out included descriptive data analysis and bivariate data analysis using logistic regression to see the relationship between age, patient susceptibility with a primary diagnosis of DM, sepsis, and major surgery with AKI mortality. 


\section{RESULTS}

\section{Characteristics of AKI Patients in the ICU of Dr. Soetomo Hospital}

Characteristics of AKI patients in the ICU of Dr. Soetomo Hospital is presented in the following table.

Table 1. Characteristics of AKI Patients in the ICU of Dr. Soetomo Hospital

\begin{tabular}{|c|c|c|}
\hline Characteristics & Frequency $(n=23)$ & Percentage \\
\hline \multicolumn{3}{|l|}{ Gender } \\
\hline Male & 19 & 82.60 \\
\hline Female & 4 & 17.39 \\
\hline Patient who died & & 30.43 \\
\hline \multicolumn{3}{|l|}{ Age (y.o) } \\
\hline $20-29$ & 4 & 17.39 \\
\hline $30-39$ & 2 & 8.69 \\
\hline $40-49$ & 2 & 8.69 \\
\hline $50-59$ & 7 & 30.43 \\
\hline $60-69$ & 5 & 21.73 \\
\hline $70-79$ & 1 & 4.34 \\
\hline $80-89$ & 2 & 8.69 \\
\hline \multicolumn{3}{|l|}{ Primary diagnosis } \\
\hline Diabetes & 8 & 34.78 \\
\hline Myocard.infark & 4 & 17.39 \\
\hline Malignancy & 3 & 12.5 \\
\hline Pulmonary edema & 3 & 12.5 \\
\hline Femur fracture & 1 & 4.34 \\
\hline Tetanus & 1 & 4.34 \\
\hline Myasthenia gravis & 1 & 4.34 \\
\hline Eclampsia & 1 & 4.34 \\
\hline Pickwickian syndrome & 1 & 4.34 \\
\hline \multicolumn{3}{|l|}{ Staging AKI (KDIGO) } \\
\hline Stage 1 & 12 & 52.17 \\
\hline Stage 2 & 4 & 17.39 \\
\hline Stage 3 & 7 & 30.43 \\
\hline \multicolumn{3}{|c|}{ AKI enforcement interval (days) } \\
\hline $0-3$ & 16 & 69.56 \\
\hline $4-6$ & 5 & 21.73 \\
\hline $7-9$ & 0 & 0 \\
\hline $10-12$ & 1 & 4.34 \\
\hline $13-15$ & 0 & 0 \\
\hline $16-18$ & 0 & 0 \\
\hline 19- 21 & 0 & 0 \\
\hline$>21$ & 1 & 4.34 \\
\hline \multicolumn{3}{|l|}{ Length of ICU stay (days) } \\
\hline $0-3$ & 0 & 0 \\
\hline $4-6$ & 10 & 43.47 \\
\hline $7-9$ & 3 & 13.04 \\
\hline $10-12$ & 3 & 13.04 \\
\hline $13-15$ & 4 & 17.39 \\
\hline $16-18$ & 1 & 13.04 \\
\hline $19-21$ & 0 & 0 \\
\hline $22-24$ & 1 & 13.04 \\
\hline $25-27$ & 0 & 0 \\
\hline $28-30$ & 1 & 13.04 \\
\hline \multicolumn{3}{|l|}{ Use of Antibiotics } \\
\hline$\leq 1$ type & 14 & 60.86 \\
\hline$>1$ types & 9 & 39.13 \\
\hline \multicolumn{3}{|l|}{ Exposures factors } \\
\hline Septic shock & 12 & 38,70 \\
\hline Major heart surgery & 6 & 19,35 \\
\hline Radio-contrast agent & 4 & 12.90 \\
\hline Nefrotoksik agent & 2 & 6.45 \\
\hline Others major surgery & 7 & 22.58 \\
\hline \multicolumn{3}{|l|}{ Comorbid } \\
\hline Elderly & 8 & 21.62 \\
\hline Diabetes mellitus & 8 & 21.62 \\
\hline Hypertension & 7 & 18.91 \\
\hline Heart disease & 6 & 16.21 \\
\hline Lung disease & 5 & 13.51 \\
\hline Cancer & 3 & 8.10 \\
\hline
\end{tabular}


Table 1 describes that of the 23 AKI cases found, the death cases totaled $30.43 \%$ ( 7 cases) of the total number of cases. The majority of patients were male (82.6\%). Most patients were between the age of 50-59 years old (30.43\%). The primary diagnosis of patients who entered the ICU was diabetes mellitus (34.78\%). Most of the patients were at stage 1 based on the AKI staging criteria (52.17\%). The highest AKI identification in ICU patients was 0-3 days as much as $69.56 \%$ and the highest average length of stay in the ICU was around 4-6 days (43.47\%).

\section{Relationship Between Primary Diagnosis of Diabetes Mellitus and Mortality of AKI in ICU}

The findings of the bivariate study in the ICU of Dr. Soetomo Hospital between the primary diagnosis of diabetes mellitus and the mortality of AKI patients are presented in the following table.

Table 2. Bivariate analysis between primary diagnosis of diabetes mellitus and mortality in AKI patients in the ICU

\begin{tabular}{ccccc}
\hline \multirow{2}{*}{ No } & \multirow{2}{*}{ Diabetes } & \multicolumn{2}{c}{ Mortality } & Fisher test \\
\cline { 3 - 5 } & & Life & Died & \\
\hline 1 & DM (+) & $1(5.6)$ & $7(2.4)$ & 0.000 \\
2 & DM (-) & $15(10.4)$ & $0(4.6)$ & \\
& Total & 16 & 7 & \\
\hline
\end{tabular}

Based on the Fisher test table above, it can be concluded that there is a significant relationship between the susceptibility of patients with the primary diagnosis of DM with the mortality of AKI patients in the ICU $(p=0.000)$.

\section{Relationship Between Sepsis Exposure and AKI Mortality in the ICU}

The results of bivariate analysis between exposure to sepsis and mortality of AKI patients in the ICU of Dr. Soetomo Hospital is presented in the following table.

Table 3. Bivariate analysis between sepsis exposure and mortality in AKI patients in the ICU

\begin{tabular}{ccccc}
\hline \multirow{2}{*}{ No } & \multirow{2}{*}{ Septic shock } & \multicolumn{2}{c}{ Mortality } & \multirow{2}{*}{ Fisher test } \\
\cline { 3 - 4 } & & Life & Death & \\
\hline 1 & Sepsis (+) & $5(8.3)$ & $7(3.7)$ & 0.005 \\
2 & Sepsis (-) & $11(7.7)$ & $0(3.3)$ & \\
& Total & 16 & 7 & \\
\hline
\end{tabular}

Based on Fisher's test table above, it can be concluded that there is a significant relationship between sepsis exposure and mortality in AKI patients in the ICU $(\mathrm{p}=0.005)$.

\section{DISCUSSION}

The high AKI case in the ICU is thought to be because most of the cases that came in the study time period were dominated by sepsis as the main exposure factor while old age and diabetes mellitus as susceptibility factors. Moreover, the ability to detect early is greatly improved, causing patients to be quickly detected and treated. The majority of AKI patients in this study were male, as many as 19 patients $(82.6 \%)$, and the rest were female. In a multicenter study in 19 hospitals in England conducted by Osterman (2008), the number of AKI patients in the ICU was dominated by men as much as $70.2 \%{ }^{(6)}$. In a study conducted by Kim (2015) on AKI patients induced by nephrotoxic contraction agents, the percentage of men was $62.5 \%$ higher than that of women ${ }^{(7)}$. Research on pediatric patients in ICU hospitals in Brazil conducted by Marcia (2017) found that the percentage of male patients was $62.74 \%$ higher than female patients ${ }^{(8)}$. Several studies indicate that men experience AKI more quickly. This is related to the levels of sex hormones which directly or indirectly affect cell activity by modulating the synthesis of various cytokines, growth factors, and vasoactive agents. The estrogen hormone is said to have a protective effect in women up to menopause. Estrogen works by influencing the formulation and signal transduction of growth factor $\beta$ and the angiotensin system. Several studies in experimental animals using selective estrogen receptor modulators have shown that the development of AKI in experimental animals can be inhibited. In general, men have a less healthy lifestyle than women which causes a higher risk of developing AKI in men than women ${ }^{(9)}$.

In this study, 7 patients $(30.43 \%)$ had the highest age range, namely $50-59$ years old. A study conducted by Han et al (2015) stated that the age group most frequently affected by AKI and coronary heart disease were elderly people 50-55 years old with an average age of 53 years old ${ }^{(10)}$. According to Germaine (2020), various diseases arise after age ${ }^{(11)}$. Osterman (2020) added that certain mutations in the DNA of his body cells, one of which is the shortened telomeres, would be faced by someone who lives a very long time. Mutation of just one DNA base from the transcription process might cause re-frame and shifting of the translation of an amino acid in the ribosome to change instead of being disturbed until it is fatal. The production of proteins such as enzymes, immune cells, and other important components is defective. Thus, disease and susceptibility begin to arise in an elderly person. In the case of the kidneys, structural and functional changes occur. The changes that occur lead to a decrease in renal mass, number of nephrons, and the function of renal hemostasis. In a healthy condition, the kidneys can adapt to hemodynamic changes. However, in pathological conditions, the old kidneys are less able to 
adapt. Moreover, other factors increase the risk of AKI such as exposure to nephrotoxic drugs, invasive procedures, and infections ${ }^{(12)}$.

Of the 23 AKI cases found, 7 cases of death or $30.43 \%$ of the total number of cases. According to a study conducted by Brown et al (2016), a meta-analysis of MMR from 2001 to 2011 showed a trend of increasing incidence of $14 \%$ with a twofold increase in mortality in the United States ${ }^{(13)}$.

There was a significant relationship between DM and MMR mortality in the ICU $(p=0.000)$ based on the findings of the statistical test study. They concluded that DM has the ability to increase the risk of AKI, according to Patschan et al. (2016) in their report, and it also increases AKI mortality and morbidity in the long term. If it is related to the susceptibility of patients who in this case are the hosts, the cause of diabetes mellitus is often called multifactorial. Factors such as genetics, decreased physiological function of pancreatic beta cells have the potential to develop into a chronic disease. Thus, they have the potential to cause both microvascular and macrovascular complications, one of which is complications to the kidneys ${ }^{(5)}$.

The statistical analysis findings showed that there was a significant relationship between septic shock and mortality in ICU patients with AKI $(\mathrm{p}=0.005)$. A study conducted by Rodrigo et al. (2017) on 405 patients with septic shock who entered the ICU room at the Marques de Valdecila, Spanyol, revealed that sepsis is the main cause of AKI and AKI occurrence also has a significant effect on AKI mortality in the ICU room $(\mathrm{p}=0.000)^{(14)}$. Several studies have suggested that it is associated with microvascular changes, dysregulation of homeostasis from infection, necrosis/apoptosis, and hypoxic-ischemic lesions ${ }^{(15)}$. When associated with the epidemiological triangle, humans who have AKI as hosts are said to be weak and vulnerable to fall into a more severe state when aggravated by factors from outside the environment. A weak host must be susceptible to infection even the slightest infection can be very difficult and take a long time to heal. When the infection goes untreated and has caused organ failure, it is considered a septic shock. According to Patschan et al (2016), there is a high mortality rate without comorbid septic shock. If it is comorbid, then of course clinicians need to be vigilant and be prepared to overcome all possibilities ${ }^{(5)}$.

\section{CONCLUSION}

AKI mortality in the ICU of Dr. Soetomo Hospital is $30.43 \%$ of the total number of cases of 23 cases. The majority of patients were male and most patients were between the age of 50-59 years old. The primary diagnosis of patients who entered the ICU was diabetes mellitus and most of the patients were at stage 1 based on the AKI staging criteria. Factors associated with AKI mortality were the primary diagnosis of DM and exposure to septic shock. Further researchers with wider scope and larger number of AKI case samples is needed to get more representative AKI cases in ICU.

\section{REFERENCES}

1. Ronco C, et al. Acute Kidney Injury. Lancet. 2019;394:1949-1964

2. Hoste EA, et al. Epidemiology of Acute Kidney Injury in Critically Ill Patient: The Multinational AKI-EPI Study. Intensive Care Med. 2015;41: 1411-1423

3. Harrois A, et al. Prevalence and Risk Factor for Acute Kidney Injury Among Trauma Patient: A Multicenter Cohort Study. Critical Care. 2018;22: 344

4. Venot M, et al. Acute Kidney Injury in Severe Sepsis and Septic Shock in Patient with and without Diabetes Mellitus: A Multicenter Study. PLOS one. 2015;10(5): 1-10

5. Patschan D, et al. Review Article Acute of Kidney Injury in Diabetes Mellitus. International Journal of Nephrology. 2016: 1-7

6. Ostermann M, et al. Renal Failure in the Intensive Care Unit: Acute Kidney Injury Compared to End-stage Renal Failure. Critical Care. 2008; 12(432): 1-2

7. Kim MH, et al. Incidence and Outcome of Contrast-associated Acute Kidney Injury Assessed with Risk, Injury, Failure, Loss, and End-stage Kidney Disease (RIFLE) Criteria in Critically Ill Patients of Medical and Surgical Intensive Care Units: A Retrospective Study. BMC Anesthesiol. 2015; 15(23): 1-8

8. Marcia C, et al. Predictive Factors of Mortality in Pediatric Patients with Acute Renal Injury Associated with Sepsis. Jornal de Pediatria. 2017; 93(1): 28-34

9. Neugarten J, Golestaneh L. Gender and the Prevalence and Progression of Renal Disease. Advances in Chronic Kidney Diseases. 2013; 20(5): 390-395

10. Han SS, et al. Effect of Acute Kidney Injury and Chronic Kidney Disease on Long Term Mortality After Coronary Artery Bypass Grafting. American Heart Journal. 2015; 169(3): 419-425

11. Wong G, Coates T. Epidemiology of Kidney Disease: Consolidating and Integrating the Evidence to Improve Kidney Care from Early Childhood to Adulthood. Kidney International. 2020; 98(6): 1378-1381

12. Osterman M, et al. Controversies in Acute Kidney Injury: Conclusions from a Kidney Disease: Improving Global Outcomes (KDIGO) Conference. Kidney International. 2020; 98(2): 294-309

13. Brown JR, et al. Hospital Mortality in the United States Following Acute Kidney Injury. BioMed Research International. 2016; 2016:1-6

14. Rodrigo E, et al. Association Between Recurrence of Acute Kidney Injury and Mortality in Intensive Care Unit Patient with Severe Sepsis. Journal of Intensive Care. 2017; 5(28): 1-8

15. Louzada CF, Ferreira AR. Evaluation of the Prevalence and Factors Associated with Acute Kidney Injury in A Pediatric Intensive Care Unit. Jornal de Pediatria. 2020; 913: 1-7 